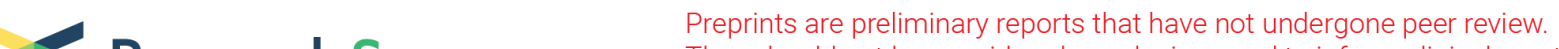 $\begin{array}{ll}\text { Research Square } & \begin{array}{l}\text { They should not be considered conclusive, used to inform clinical practice, } \\ \text { or referenced by the media as validated information. }\end{array}\end{array}$
}

\section{Mass spectrometric analysis of active ingredients in fertilized egg for lipid metabolism}

pei Ma

shanxi agriculture university https://orcid.org/0000-0002-9766-6313

yilei Li

Shanxi Agricultural University

pei Wen

Shanxi Agricultural University

yufang $\mathrm{Ge}$

Shanxi Agricultural University

ding Yang

Shanxi Agricultural University

jiahui Yuan

Shanxi Agricultural University

pengxiang Zhang

Shanxi Agricultural University

zhiwei Zhu

Shanxi Agricultural University

xiaomao Luo

Shanxi Agricultural University

haidong Wang ( $\checkmark$ wanghaidong@sxau.edu.cn )

https://orcid.org/0000-0002-2762-0600

\section{Research article}

Keywords: fertilized egg, mass spectrometry, lipid metabolism

Posted Date: January 29th, 2020

DOI: https://doi.org/10.21203/rs.2.22228/v1

License: (9) This work is licensed under a Creative Commons Attribution 4.0 International License. Read Full License 


\title{
Mass spectrometric analysis of active ingredients in fertilized egg for lipid metabolism
}

\author{
PEI MA ${ }^{1}$, YILEI LI ${ }^{1}$, PEI WEN ${ }^{1}$, YUFANG GE ${ }^{1}$, DING YANG ${ }^{1}$, JIAHUI YUAN ${ }^{1}$, \\ PENGXIANG ZHANG ${ }^{1}$, ZHIWEI ZHU $^{2}$, XIAOMAO LUO $^{1}$, HAIDONG WANG $^{1 *}$
}

${ }^{1}$ College of Animal Science and Veterinary Medicine, Shanxi Agricultural University, Jinzhong, Shanxi 030801, P.R. China;

${ }^{2}$ College of Life Science, Shanxi Agricultural University, Jinzhong, Shanxi 030801, P.R. China.

*Correspondence to: Prof. Haidong Wang, College of Animal Science and Veterinary Medicine, Shanxi Agricultural University, No.1 Mingxian South Road, Jinzhong, Shanxi 030801, P.R. China, mail: wanghaidong@sxau.edu.cn

Running title: Active ingredients analysis in fertilized egg

\begin{abstract}
Adipose tissue is one of the important components of the animal body, and its development is restricted by various mechanisms. Yolk protein has an important effect on the development of chicken embryo skeletal muscle. The protein needed for chicken embryo development mainly comes from egg yolk. The protein metabolism in yolk is particularly strong after fertilization, and the nutrition level is much higher than that of ordinary eggs. In order to study which components in fertilized eggs play a role in fat metabolism, Nano-HPLC-MS/MS protein detection was performed on fertilized egg extracts. A total of 295 proteins were identified and analyzed by Gene Ontology (GO), Kyoto Encyclopedia of Genes and Genomes (KEGG), and eukaryotic cluster (KOG)/homologous cluster (COG) analysis. The results indicate that metabolic pathways and PPAR signaling pathways are the main biological processes affecting lipid metabolism. Western Blotting (WB) was used to verify the possible components of fat metabolismrelated genes apolipo A-I (APOA1) and bile salt-activated lipase (BSAL, CEL). The results showed that APOA1 was highly expressed in fertilized eggs compared to unfertilized eggs. The expression of CEL was not significantly different between fertilized and unfertilized eggs. This study provides a theoretical basis for further studying the effects of fertilized eggs on biological metabolism.
\end{abstract}

Keywords: fertilized egg; mass spectrometry; lipid metabolism 


\section{Background}

The protein required for chick embryo development is mainly derived from egg yolk. After fertilization, sperm and gametes combine to form a new individuals [1] and at the same time, the protein metabolism in egg yolk is very strong,during this period, the content of protein, total amino acids, and free amino acids gradually increased after fertilization, and the nutritional level was much higher than that of ordinary eggs [2] and biological activities supporting new life development [3]. In the early days, proteomics based on high-throughput mass spectrometry greatly facilitated our understanding of the quantity and properties of the constituent proteins in the analysis of eggs [4]. In previous studies, mass spectrometry was used to study LDL in egg yolk, which provided a basis for the study of egg yolk protein composition [5]. TMT-labeled tryptic digest and mass spectrometry were performed on the yolks of fertilized eggs, and 225 proteins were detected. Nine proteins were enriched in the yolk of fertilized eggs compared to the yolk of unfertilized eggs. On this basis, the yolk of fertilized eggs is used to enrich or isolate proteins with pro-angiogenic and antimicrobial properties [6]. Analysis of fertilized and unfertilized eggs using 2-dimensional gel electrophoresis (2-DE), coupled with matrix-assisted laser desorption/ionization time-of-flight tandem mass spectrometry (MALDI-TOF MS/MS), the expression of ovalbumin family is higher in fertilized eggs than in unfertilized eggs, suggesting that ovalbumin may play a significant role in embryo development [7]. It has also been reported that the protein content of eggshell membrane in fertilized eggs varies with the incubation time. After identifying eggshell membrane, a total of 228 eggshell membrane proteins were found [8]. Zhang [9] research found that a total of 1241 proteins were identified in fertilized eggs and unfertilized eggs. Among them, there were 229 protein expression differences between fertilized eggs and unfertilized eggs ( $\mathrm{p}<0.05$ ), of which 86 proteins were upregulated and 48 proteins were down-regulated.

With the changes in the lifestyle of modern society and the frequent consumption of high-fat and high-sugar foods, the proportion of obese people has gradually increased. This has caused diseases such as diabetes, hyperlipidemia and hypertension [10]. Being able to use meals to change fat accumulation has become a hot spot. This knowledge can be used to develop fertilized egg-based products for specific applications. The mechanism of adipogenesis is regulated by a number of transcription factors, such as peroxisome proliferation-activated receptor-receptor (PPAR) and CCAAT/enhancer binding protein (C/EBP) [11].

Whitehead [12] found that fat cannot enter cells in unhydrolyzed form, so lipolysis becomes particularly important for general metabolism.Lipidtoxicity induces insulin resistance in nonfatty tissues and organs such as liver and muscles, like novel protein kinase c pathways and the JNK1 pathway [13]. Depending on the cell or tissue type, autophagy may play a role in lipid metabolism. In the case of high-fat diets or long-term fasting, autophagy can lead to lipolysis. In a normal diet, autophagy seems to be involved in adipocyte differentiation and adipogenesis, but not in lipolysis [14]. In addition, adenosine monophosphate-activated protein kinase (AMPK) is expressed in fat, liver, and skeletal muscle [15], it is also an important regulator of lipid metabolism and can increase fatty acid oxidation and reduce lipid synthesis [16].

In previous studies, proteomics of fertilized eggs has been studied, but the specific protein analysis is incomplete. Therefore, this study will obtain systematic data on proteomics of fertilized eggs, so as to provide a basis for future studies on fertilized eggs. Lipid metabolism plays a very important role in life activities, after mass spectrometry analysis of fertilized eggs, we screened out genes related to Lipid metabolism and verified by western blot. 


\section{Result}

\section{GO functional annotation analysis of proteomic differences}

GO classification of the identified proteins, the detected proteins are annotated as biological processes, molecular functions, and cellular components, each picking the top 20 pathways with the largest number of proteins (Figure 1). Reference website http://www.geneontology.org. The most highly enriched biological process was signal transduction (GO:0007165), proteolysis (GO: 0006508) and lipid transport (GO:0006869) were the most highly enriched, containing 18,10 and 19 proteins, respectively. Protein binding (GO:0005515) and ATP binding (GO:0016020) were the most highly enriched molecular functions, containing 43 and 19 proteins, respectively. The main cellular components of these extracellular space (GO:0005615), extracellular exosome (GO:0070062) and extracellular region (GO:0005576) involving 43, 42 and 33 proteins, respectively.

\section{KEGG pathway annotation of proteomic differences}

For KEGG enrichment, differentially accumulated proteins expressed as the highest expression of Regulation of actin cytoskeleton ( 8 proteins), followed by Metabolic pathways (6 proteins) and Focal adhesion (6 proteins), followed by Endocytosis (4 proteins) and ECM-receptor interaction (4 proteins), and finally Glycolysis/Gluconeogenesis, Carbon metabolism, Biosynthesis of amino acids, PPAR signaling pathway and MAPK signaling pathway each contain 3 proteins (Figure 2, Table1). KOG based on sequence similarity-based functional classification annotation and prediction of proteomic differences

KOG analysis was performed on the differential proteins of fertilized eggs, and the top 20 groups with the highest sequence similarity were selected for annotation (Figure 3). The five largest categories are signal transduction mechanisms (33 genes), general function prediction only (31 genes), defense mechanisms (27 genes), posttranslational modification, protein turnover, chaperones (22 genes), extracellular structures (18 Genes).

\section{Verification of the results of mass spectrometry analysis of fertilized eggs}

The treated egg yolk was first subjected to gradient dilution, and each diluted gradient of egg yolk was subjected to SDS-PAGE electrophoresis, and then the gel was stained with Coomassie Brilliant blue G-250. The results showed a significant towing in the display $10^{-1}$, the $10^{-2}$ protein band was the most obvious, and the $10^{-3}, 10^{-4}, 10^{-5}$ protein bands were not obvious (Figure 4A). Thus, we chose a protein of $10^{-2}$ for the next experiment.

In order to investigate changes in the composition of fertilized and unfertilized eggs during lipid metabolism, genes APOA1 (apolipo A-I) and CEL (bile salt-activated lipase) which inhibit Lipid development were selected. The results showed that APOA1 was significantly higher in fertilized eggs than in unfertilized eggs. It indicates that APOA1 has a lipid-lowering effect in fertilized eggs. The difference in expression of CEL between fertilized and unfertilized eggs was not significant (Figure 4B and 4C).

\section{Discussion}

After mass spectrometry of the fertilized egg, the differentially expressed genes screened reflect the biological function of the fertilized egg in different physiological processes by the distribution in the GO analysis. There are differentially expressed genes in biological processes, cell components and molecular functions. The differential gene distribution in cell components is the most, and the differential gene distribution is the least in biological processes. The cellular 
components that significantly enrich the differential genes are mainly extracellular space, extracellular exosome, extracellular region, and integral component of membrane. The differential genes are significantly enriched in molecular functions such as protein binding, ATP binding, calciumion binding, serine-type endopeptidase inhibitor activity. In terms of biological processes, it is mainly enriched in signal transduction, proteolysis, and lipid transport.

Adipose tissue is the largest reservoir of energy in mammals and is considered to be a dynamic organ of complex decomposition and anabolism. There are two main types, white adipose tissue (WAT) and brown adipose tissue (BAT), White adipose tissue is the primary site for fat storage, while brown adipose has the ability to produce heat without trembling. Adipose tissue also secretes a variety of important endocrine hormones, such as leptin, adiponectin, and resistin. It is important to study the development mechanism of adipose tissue regulation to improve metabolic disease and meat quality. PPAR $\alpha$ plays an important role in lipid and sugar metabolism, adipocyte differentiation, oxidation of fatty acids, and inflammation [17]. Activation of PPAR $\alpha$ can lead to cellular fatty acid absorption and lipoprotein metabolism, which in turn reduces triglyceride levels and increases HDL cholesterol levels [18]. Apolipoprotein A-I (APOA1), which is the major protein component of high density lipoprotein (HDL) in plasma and a key component in regulating lipoprotein metabolism and cardiovascular disease risk [19] APOA1 is the trigger of BAT without shaking to produce heat [20]. Decreased nuclear receptor PPARr in inguinal fat in aging mice can increase adipose tissue expansion and insulin resistance. These metabolic effects are accompanied by reduced heat production, reduced brown fat gene levels, and browning of subcutaneous adipose tissue [21]. Perilipin-1 (Plin1) are located on the surface of adipocyte lipid droplets and regulate the storage and hydrolysis of fatty triglycerides. In obesity, reduced levels of perilipin in adipocytes, leading to a lower rate of lipolysis. Compared with WT mice, perilipin transgenic mice have reduced white fat and weight in perilipin transgenic mice, indicating that increased perilipin gene expression can prevent diet-induced obesity [22]. The results of WB showed that the expression of APOA1 in fertilized eggs was higher than that in unfertilized eggs, suggesting that APOAI in fertilized eggs was more conducive to the browning of fat than in unfertilized eggs. Bile salt-activated lipase, also known as carboxyl ester lipase (CEL), a multiple function lipolytic enzyme [23]. In early studies, bile salt-activated lipase were given to milk-based dietary ingredients to activate lipase to enhance fat digestion and thereby increase growth rate [24]. Today, studies have found that CEL can improve patients with pancreatic insufficiency and fat malabsorption caused by pathological reasons [25].

\section{Conclusions}

The differential protein enrichment of fertilized eggs by HPLC analysis can provide a solid theoretical basis for the subsequent study of fertilized eggs. In addition, after WB validated APOA1, CEL related to lipid metabolism, we found that the expression of APOA1 in fertilized egg was higher than that in unfertilized egg, while the difference between CEL in fertilized egg and unfertilized egg was not significant. Therefore, we suspect that the presence of APOA1 protein in fertilized eggs can inhibit white fat formation and promote brown fat formation (Figure 5).

\section{Methods}

\section{Treatment of Sample}

Take a fertilized egg, use a glass rod to knock a small hole in the eggshell, let the egg white flow out of the small hole, separate the whole egg yolk, use the needle to pick up the egg yolk capsule, and carefully collect the egg yolk in the centrifuge tube with a syringe. Add PBS (yolk: 
PBS $=7: 3$ ) to the centrifuge tube, centrifuge the egg yolk suspension at $3340 \mathrm{~g}$ for 20 min at $4{ }^{\circ} \mathrm{C}$, collect the precipitate, and store at $4^{\circ} \mathrm{C}$.

\section{Mass spectrometry analysis}

Sample prepatation and In-gel digestion

Each sample was allowed to proceed at $56^{\circ} \mathrm{C}$ for $1 \mathrm{~h}$ in an appropriate volume of $10 \mathrm{~mm}$ dithiotreitol in $25 \mathrm{mM} \mathrm{NH}_{4} \mathrm{HCO}_{3}$ and the alkylation was allowed to proceed in the dark for $45 \mathrm{~min}$ at room temperature in an appropriate volume of $55 \mathrm{mM}$ iodoacetamide in $25 \mathrm{mM} \mathrm{NH} \mathrm{HCO}_{3}$. The gel pieces were washed with $100 \mu \mathrm{L}$ of $25 \mathrm{mM} \mathrm{NH}_{4} \mathrm{HCO}_{3}$ for $10 \mathrm{~min}$ and dehydrated with $100 \mu \mathrm{L}$ of $25 \mathrm{mM} \mathrm{NH} \mathrm{HCO}_{3}$ in $50 \%$ acetonitrile for $5 \mathrm{~min}$. The wash and dehydration step was repeated once. Following drying in a SpeedVac, the gel pieces were mixed with an appropriate volume of $12.5 \mathrm{ng} / \mu \mathrm{l}$ of trypsin and incubated on ice for $40 \mathrm{~min}$ and $25 \mathrm{mM} \mathrm{NH}_{4} \mathrm{HCO}_{3}$ was added as needed to cover the gel pieces. Digestion was then carried out at $37^{\circ} \mathrm{C}$ overnight. To extract the tryptic peptides from the gel pieces, an appropriate volume of $60 \%$ acetonitrile, $0.2 \%$ TFA, was added. Following $20 \mathrm{~min}$ of vortex and $5 \mathrm{~min}$ of sonication, the supernatant was taken and saved. Following the evaporation of acetonitrile in a SpeedVac, the sample was desalted with a C18 ZipTip (Millipore), and half of the eluate was analyzed with nano-LC-MS/MS.

Nano-HPLC-MS/MS analysis

The samples were resuspended with $30 \mu \mathrm{L}$ solvent $\mathrm{C}$ respectively $(\mathrm{C}$ : water with $0.1 \%$ formic acid), separated by nanoLC and analyzed by on-line electrospray tandem mass spectrometry. The experiments were performed on a Nano ACQUITY UPLC system (Waters Corporation, Milford, MA) connected to a Q-Exactive mass spectrometer (Thermo Fisher Scientific, MA, USA) equipped with an online nano-electrospray ion source. $10 \mu \mathrm{L}$ peptide sample was loaded onto the trap column (Thermo Scientific Acclaim PepMap C18, $100 \mu \mathrm{m} \times 2 \mathrm{~cm}$ ), with a flow of $10 \mu \mathrm{L} / \mathrm{min}$ for 3 min and subsequently separated on the analytical column (Acclaim PepMap C18, $75 \mu \mathrm{m} \times 15 \mathrm{~cm}$ ) with a 90min linear gradient, from $5 \% \mathrm{D}$ (D:ACN with $0.1 \%$ formic acid) to $55 \% \mathrm{D}$. The column was reequilibrated at initial conditions for $10 \mathrm{~min}$. The column flow rate was maintained at $300 \mathrm{~nL} / \mathrm{min}$. The electrospray voltage of $2 \mathrm{kV}$ versus the inlet of the mass spectrometer was used.

The mass spectrometer was run under data dependent acquisition mode, and automatically switched under MS and MS/MS mode. MS1 mass resolution was set as $70 \mathrm{~K}$ with m/z 300-1800 and MS/MS resolution was set as $17.5 \mathrm{~K}$ under HCD mode. The dynamic exclusion time was set as $10 \mathrm{sec}$.

Database Searching Parameters

Tandem mass spectra were processed by PEAKS Studio version 8.5 (Bioinformatics Solutions Inc., Waterloo, Canada). PEAKS DB was set up to search the UniProt-gallus gallus database (ver 201708, 29725 entries) assuming trypsin as the digestion enzyme. PEAKS DB were searched with a fragment ion mass tolerance of $0.05 \mathrm{Da}$ and a parent ion tolerance of $7 \mathrm{ppm}$. Carbamidomethylation (C) was specified as the fixed modification. Oxidation (M), Deamidation (NQ), and Acetylation (Protein N-term), were specified as the variable modifications. Peptides were filter by $1 \%$ FDR and 1 unique peptide.

Blast2GO version 4 was used for functional annotation. Whole protein sequence database was analyzed by BlastP using whole database and mapped, annotated with gene ontology database. Statistically altered functions of different expressed proteins was calculated by Fisher's exact test in BLAST2GO(P<0.05).Pathway analysis was processed by KOBAS (http://kobas.cbi.pku.edu. cn/) COG\&KOG Analysis was based on the Phylogenetic classification of proteins encoded in complete 
genomes (NCBI, www.ncbi.nlm.nih.gov/COG/) project.

\section{SDS-PAGE}

Gradient dilution of the above samples with PBS. The total protein loading quantity of each sample was $30 \mathrm{ug}$ and heated at $95^{\circ} \mathrm{C}$ for $10 \mathrm{~min}$. Each gradient diluted sample was subjected to SDS-PAGE electrophoresis and run at $80 \mathrm{~V}$ until the samples reached the front of the gel. Gels were stained using Coomassie Brilliant blue G-250 (Solarbio, Beijing, China). Images were acquired by the Gel Doc XR imaging system and analyzed by software Imgae lab (All Bio-Rad, California, America).

\section{Western Blot}

The preparation of SDS-PAGE electrophoresis is the same as 2.3, the sample was transferred to the nitrocellulose membrane (BOSTER Biotechnology Co. Ltd, Wuhan, China). The membranes were blocked with 5\% non-fat dried milk in Tris-buffered saline- Tween (TBST) for $1 \mathrm{~h}$. The rabbit polyclonal antibodies include CEL (Bioss Biotech CO., Ltd.; Beijing, China, cat. no. bs-13830R, 1:1000), APOA1 (Bioss Biotech CO., Ltd.; Beijing, China, cat. no. bs-0849R, 1:1000) and the rabbit monoclonal antibody GAPDH (Proteintech Group, Inc.; cat. no. 60004-1-lg, 1:2000) primary antibodies were added for incubation overnight at $4^{\circ} \mathrm{C}$. The sample was shaken and washed three times with TBST (10 min each), and then incubated with horseradish peroxidase-conjugated secondary antibodies (ComWin Biotech Co., Ltd.; Beijing, China, CW0156 1:10,000) for $1 \mathrm{~h}$ at $37^{\circ} \mathrm{C}$ thermostatic Shaker. Then shaken and washed six times with TBST (5 min each). Finally, immunoreactive signals were developed using ECL Western blot detection reagents (ComWin Biotech Co., Ltd.; cat. no. CW0049S).

\section{Statistic methods}

The data were presented as means \pm SEMs (SD). SPSS 17.0 software (IBM, Armonk, NY, USA) was used to statistical analysis. Data of multiple groups were analyzed using One-Way ANOVA followed by Bonferroni post-hoc test, while comparisons between 2 groups were performed by unpaired Student's test. Statistical significance was defined as $\mathrm{P}<0.05$.

\section{Acknowledgements}

The present study was sponsored by Key R\&D Projects in Shanxi Province (No. 201803D221022-2, 201803D31062) , the National Natural Science Foundation of China (grant no. 31772690), the special fund for the central government guides local science and technology development (grant no. YDZX20181400004357), Scientific and Technological Innovation Project for Excellent Talents in Shanxi Province(No. 201805D211012) and the Fund for Shanxi "1331 Project" Key Innovative Research Team. All authors read and approved the final manuscript.

\section{Compenting interests}

The authors declare that they have no competing interests. 


\section{References}

1. Machaty Z, Miller AR, Lu Z. Egg activation at fertilization. Springer,Cham. 2017,953:1-47.

2. Zhang Y, LI F, Wang LS. Changes of nutrient in chicken embryo and eggs during the incubation period. Journal of Northeast Agricultural University. 2010,3.

3. Wang J, Wu J. Proteomic analysis of fertilized egg white during early incubation. EuPA Open Proteomics. 2014,2:38-59.

4. Mann K, Olsen JV, Maček B, Gnad F, Mann M. Identification of new chicken egg proteins by mass spectrometry-based proteomic analysis. World's poultry science journal. 2008;64(2):209218.

5. Jolivet P, Boulard C, Beaumal V, et al. Protein components of low-density lipoproteins purified from hen egg yolk. Journal of agricultural and food chemistry. 2006;54(12):4424-4429.

6. Padliya ND, Qian M, Roy SM, et al. The impact of fertilization on the chicken egg yolk plasma and granule proteome 24 hours post-lay at room temperature: capitalizing on high-pH/low-pH reverse phase chromatography in conjunction with tandem mass tag (TMT) technology. Food \& function. 2015;6(7): 2303-2314.

7. Qiu N, Liu W, Ma M, Zhao L, Li Y. Differences between fertilized and unfertilized chicken egg white proteins revealed by 2-dimensional gel electrophoresis-based proteomic analysis. Poultry science. 2013;92(3):782-786.

8. Cordeiro CM, Hincke MT. Quantitative proteomics analysis of eggshell membrane proteins during chick embryonic development. Journal of proteomics. 2016;130:11-25.

9. Zhang L, Chen J, Fan B, Fu M, Sun Y, Wang Y, Wang F. Label-free proteomic analysis reveals the differentiation between unfertilized and fertilized Beijing-You chicken eggs. International journal of biological macromolecules. 2019.

10. Guan YQ, liu J, noorbi nur mai mai. Mechanism and research progress of Browning white fat. Journal of xinjiang medical university. 2019(9).

11. Yeh W C, Cao Z, Classon M. Cascade regulation of terminal adipocyte differentiation by three members of the C/EBP family of leucine zipper proteins. Genes \& Development. 1995; $9(2): 168-181$.

12. Whitehead R H. A note on the absorption of fat. American Journal of Physiology-Legacy Content. 1909;24(2):294-296.

13. Sezer, Havva. Insulin resistance, obesity and lipotoxicity. Obesity and Lipotoxicity. Springer, Cham. 2017,960:277-304.

14. Zechner R, Zimmermann R, Eichmann TO, Kohlwein SD, Haemmerle G, Lass A, Madeo F. FAT SIGNALS-lipases and lipolysis in lipid metabolism and signaling. Cell metabolism. 2012;15(3):279-291.

15. Hardie D G, Scott JW, Pan DA, et al. Management of cellular energy by the AMP - activated protein kinase system. FEBS letters. 2003;546(1):113-120.

16. Kohjima M, Higuchi N, Kato M, et al. SREBP-1c, regulated by the insulin and AMPK signaling pathways, plays a role in nonalcoholic fatty liver disease. International Journal of Molecular Medicine. 2008;21(4):507-511.

17. Corton JC, Cunningham ML, Hummer BT, et al. Mode of action framework analysis for receptor-mediated toxicity: The peroxisome proliferator-activated receptor alpha (PPAR $\alpha$ ) as a case study. Critical reviews in toxicology. 2014;44(1):1-49.

18. Ferré P. The biology of peroxisome proliferator-activated receptors: relationship with lipid 
metabolism and insulin sensitivity. Diabetes. 2004;53(suppl 1): S43-S50.

19. Chao-Qiang, Lai, Laurence D, Parnell, Jose M, Ordovas. The APOA1/C3/A4/A5 gene cluster, lipid metabolism and cardiovascular disease risk. Current opinion in lipidology. 2005;16(2):153-66.

20. Kalogeropoulou C, Hatziri A, Xepapadaki E, et al. Isoform and tissue dependent impact of apolipoprotein $\mathrm{E}$ on adipose tissue metabolic activation: The role of apolipoprotein A1. Biochimica et Biophysica Acta (BBA)-Molecular and Cell Biology of Lipids. 2020;1865(2): 158551.

21. Xu L, Ma X, Verma NK, et al. Ablation of PPAR $\gamma$ in subcutaneous fat exacerbates ageassociated obesity and metabolic decline. Aging cell. 2018;17(2):12721.

22. Bae JY, Woo J, Roh HT, et al. The effects of detraining and training on adipose tissue lipid droplet in obese mice after chronic high-fat diet. Lipids in Health and Disease. 2017;16(1):13.

23. Wang CS, Hartsuck JA. Bile salt-activated lipase. A multiple function lipolytic enzyme. Biochimica et Biophysica Acta (BBA)-Lipids and Lipid Metabolism. 1993;1166(1):1-19.

24. Tang JJN, Wang CS. Dietary compositions and methods using bile salt-activated lipase. 1990.

25. Wang Y, Ding F, Wang T, et al. Purification and characterization of recombinant human bile saltstimulated lipase expressed in milk of transgenic cloned cows. PloS one. 2017;12(5): e0176864. 


\section{Figure legend}

Figure 1. GO classification results can be visualized using Bar graphs. The GO classification Bar map plots the top 20 pathways with the highest number of proteins. The ordinate indicates the percentage of total protein in each secondary classification. The color indicates different primary classifications. Biological Process (Green), Molecular Function (red), Cellular Component (blue). Figure 2. The KEGG pathway annotation results are visualized using the Bar graph. The KEGG classification Bar plots the top 20 pathways with the highest number of proteins, and the ordinate indicates the percentage of protein in the total protein.

Figure 3. KOG database was used to annotate and predict the functional classification of differentially related proteins based on sequence similarity, and the functional classification of differentially related proteins was presented by column diagram. Different colors represented various KOG classifications, and the longitudinal axis indicated the number of proteins belonging to this category.

Figure 4. Validation of APOA1 and CEL proteins in fertilized eggs. (A) Total protein analysis of fertilized egg. Mr: molecular mass ladder. Samples: Gradient diluted fertilized egg. Staining with Coomassie blue G50. (B-C) Differential expression of APOA1 and CEL proteins in unfertilized and fertilized eggs by western blot. The results are shown as the mean $\pm \mathrm{SD}, \mathrm{n}=3, * * \mathrm{P}<0.01$.

Figure 5. The pattern of APOA1 promoting white fat browning in fertilized eggs

Table 1. Genes contained in the top ten pathways with the highest expression in the KEGG pathway.

\begin{tabular}{|c|c|c|}
\hline Pathway.Name & Pathway.ID & Gene.Name \\
\hline $\begin{array}{l}\text { Regulation of actin } \\
\text { cytoskeleton }\end{array}$ & gga04810 & $\begin{array}{l}\text { F2, GSN, GSN, ACTG1, FN1, ARHGEF12, } \\
\text { ITGA2, IQGAP1 }\end{array}$ \\
\hline Metabolic pathways & gga01100 & GNS, GAPDH, ENO2, PFKL, CEL, ACAC \\
\hline Focal adhesion & gga04510 & VTN, ACTG1, FN1, ITGA2, MET, COL4A6 \\
\hline Endocytosis & gga04144 & RBP, VPS37A, MET, GBF1 \\
\hline ECM-receptor interaction & gga04512 & VTN, FN1, ITGA2, COL4A6 \\
\hline Glycolysis/Gluconeogenesis & gga00010 & GAPDH, ENO2, PFKL \\
\hline Carbon metabolism & gga01200 & GAPDH, ENO2, PFKL \\
\hline Biosynthesis of amino acids & $\operatorname{gga} 01230$ & GAPDH, ENO2, PFKL \\
\hline PPAR signaling pathway & gga03320 & APOA1, APOC3, PLIN1 \\
\hline MAPK signaling pathway & gga04010 & NGF, CACNA1E, NRK \\
\hline
\end{tabular}


Figures



Figure 1

GO classification results can be visualized using Bar graphs. The GO classification Bar map plots the top 20 pathways with the highest number of proteins. The ordinate indicates the percentage of total protein in each secondary classification. The color indicates different primary classifications. Biological Process (Green), Molecular Fun ction (red), Cellular Component (blue). 


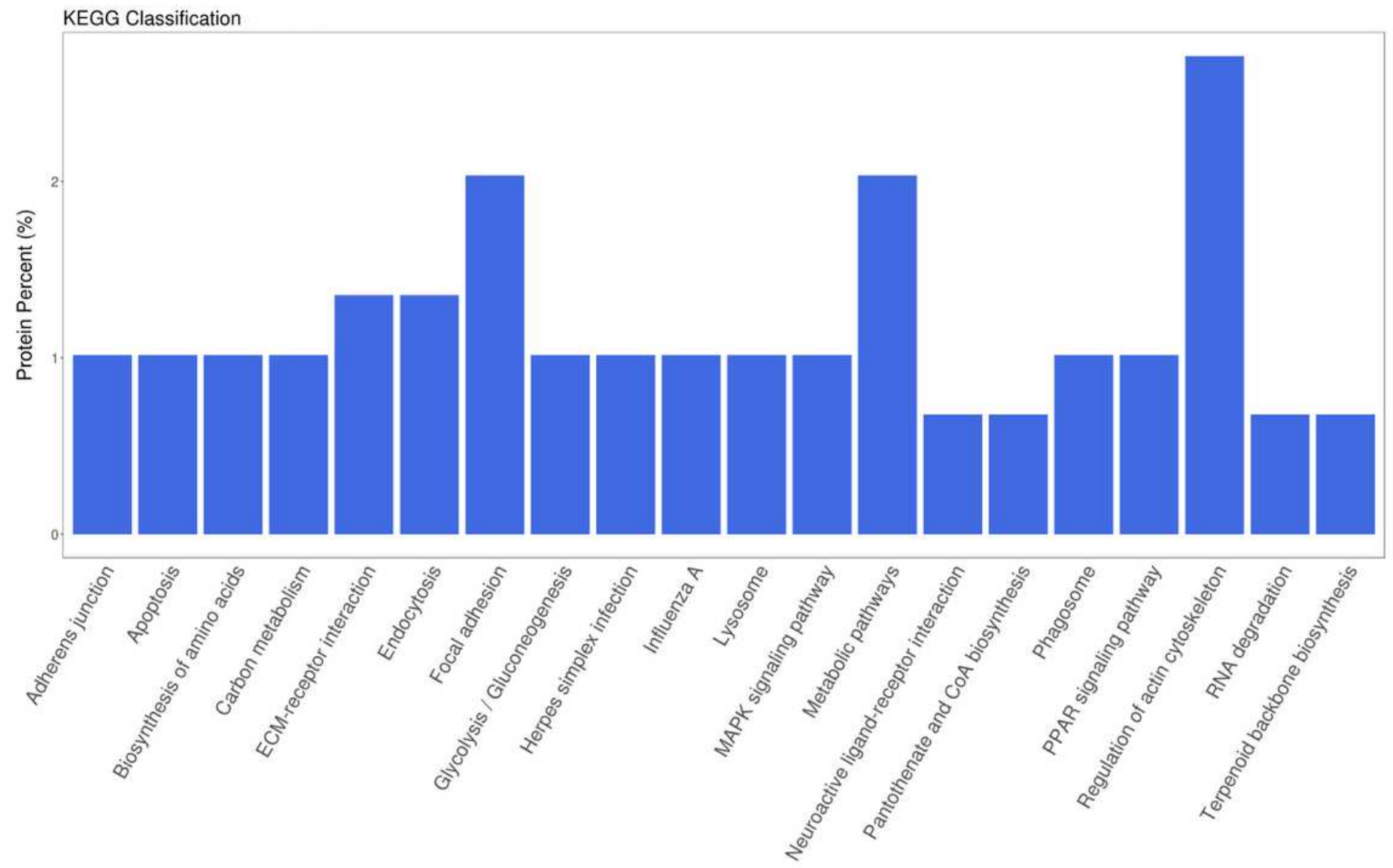

Figure 2

2. The KEGG pathway annotation results are visualized using the Bar graph. The KEGG classification Bar plots the top 20 pathways with the highest number of proteins, and the ordinate indicates the percentage of protein in the total protein. 


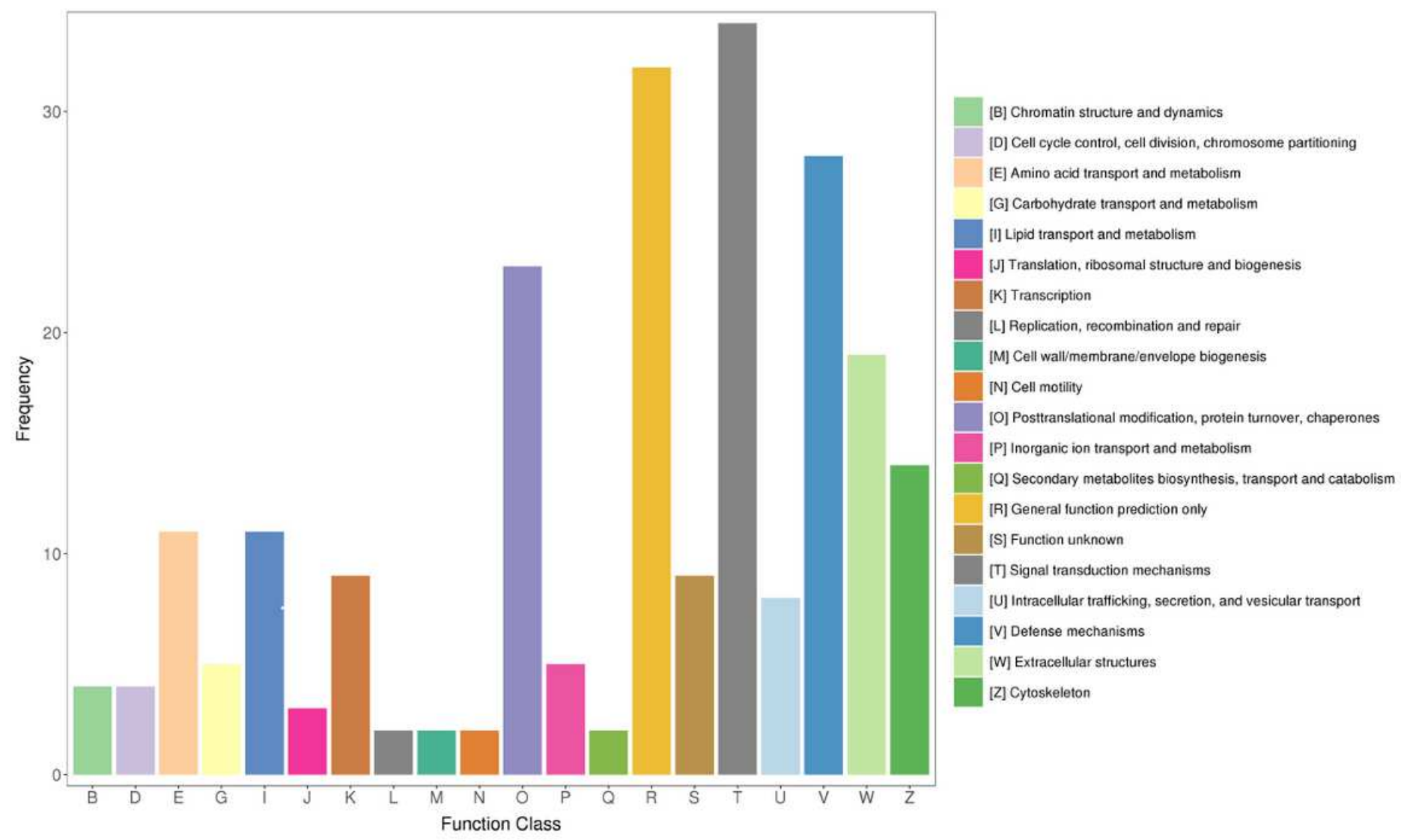

\section{Figure 3}

KOG database was used to annotate and predict the functional classification of differentially related proteins based on sequence similarity, and the functional classification of differentially related proteins was presented by column diagram. Different colors represented various KOG classifications, and the longitudinal axis indicated the number of proteins belonging to this category. 
A

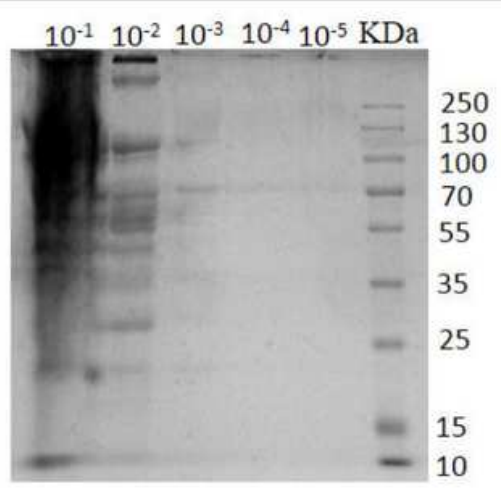

B

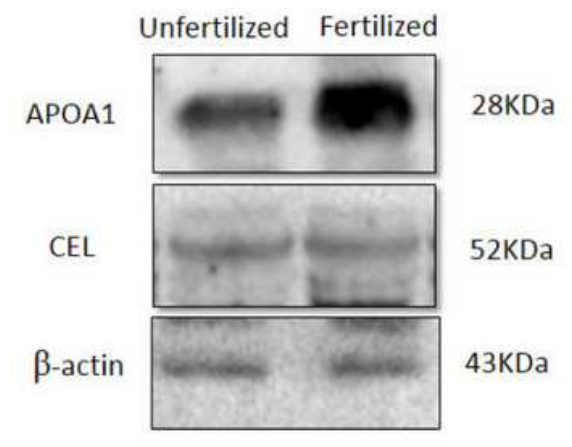

c
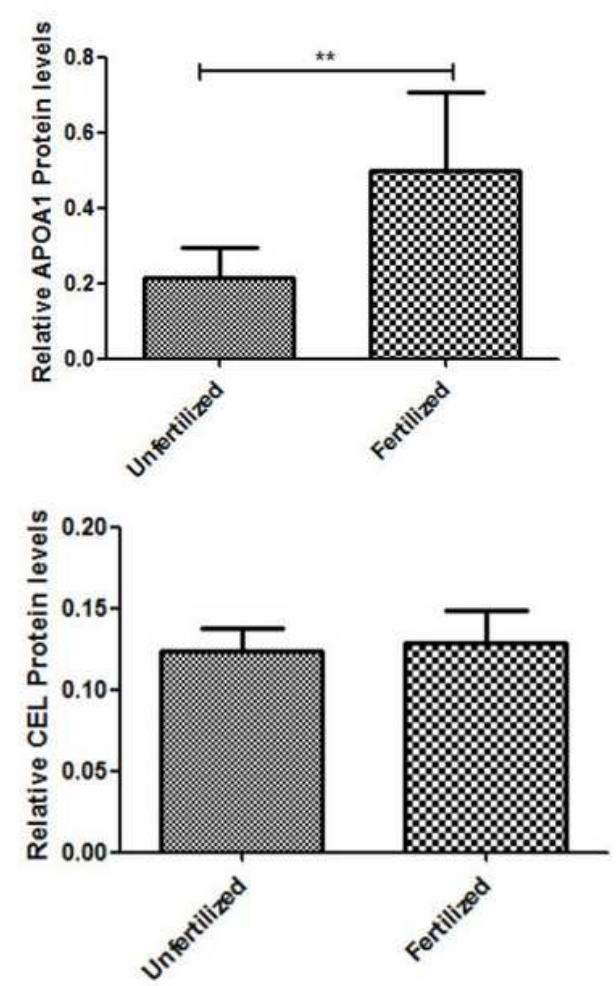

\section{Figure 4}

Validation of APOA1 and CEL proteins in fertilized eggs (A) Total protein analysis of fertilized egg. Mr: molecular mass ladder. Samples: Gradient diluted fertilized egg. Staining with Coomassie blue G50. (B C $D$ ifferential expression of APOA1 and CEL proteins in unfertilized and fertilized eggs by western blot . The resu lts are shown as the mean $\pm S D, n=3, \star \star P<0.01$. 


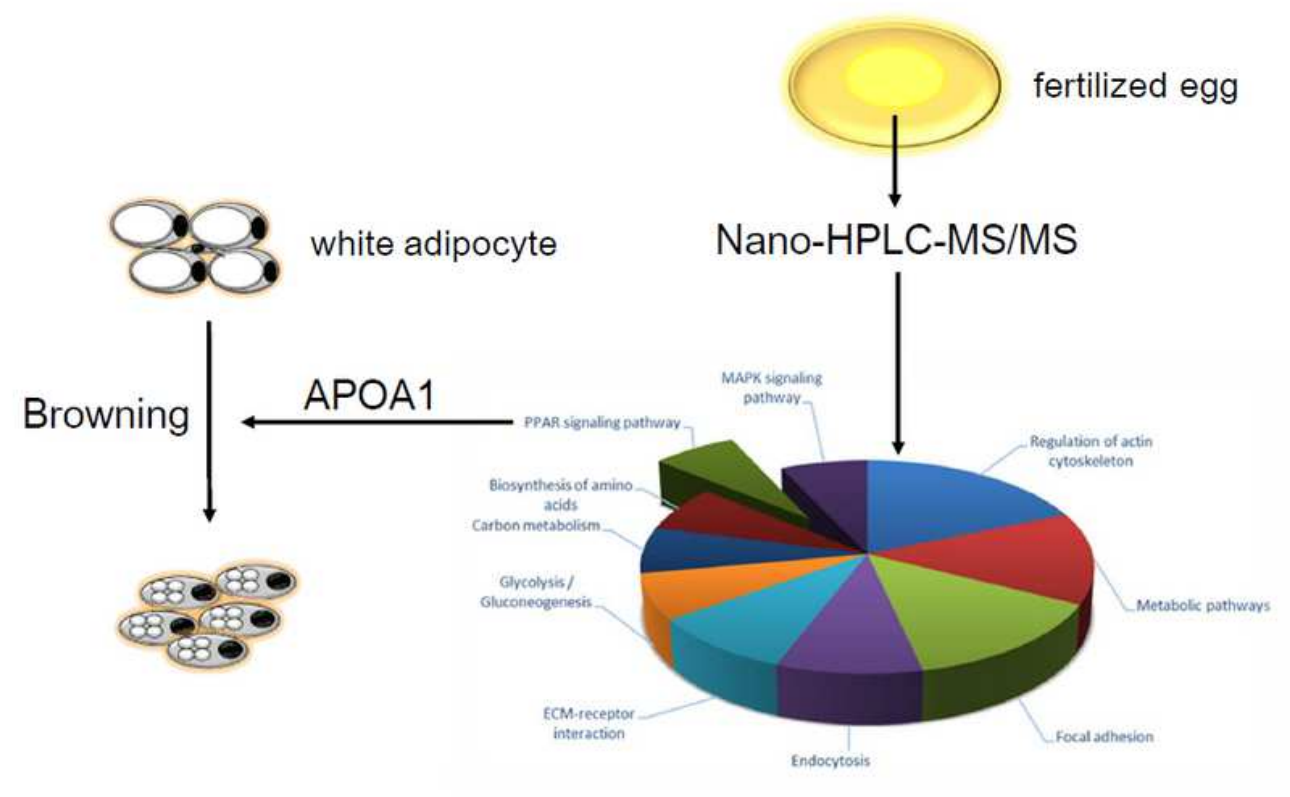

\section{Figure 5}

The pattern of APOA1 promoting white fat browning in fertilized eggs Table 1. Genes contained in the top ten pathways with the highest expression in the KEGG pathway.

\section{Supplementary Files}

This is a list of supplementary files associated with this preprint. Click to download.

- Table1.pdf 